\title{
RESEARCH
}

Open Access

\section{Prognostic factors in pulmonary metastasectomy and efficacy of repeat pulmonary metastasectomy from colorectal cancer}

Masahiro Fukada', Nobuhisa Matsuhashi ${ }^{1}$, Takao Takahashi', Yoshihiro Tanaka', Naoki Okumura', Hirotaka Yamamoto ${ }^{2}$, Koyo Shirahashi ${ }^{2}$, Hisashi Iwata ${ }^{2}$, Kiyoshi Doi ${ }^{2}$ and Kazuhiro Yoshida ${ }^{{ }^{*}}$

\begin{abstract}
Background: The rate of pulmonary metastasectomy from colorectal cancer (CRC) has increased with recent advances in chemotherapy, diagnostic techniques, and surgical procedures. The purpose of this study was to investigate the prognostic factors for response to pulmonary metastasectomy and the efficacy of repeat pulmonary metastasectomy.

Methods: This study was a retrospective, single-institution study of 126 CRC patients who underwent pulmonary metastasectomy between 2000 and 2019 at the Gifu University Hospital.

Results: The 3- and 5-year survival rates were $84.9 \%$ and 60.8\%, respectively. Among the 126 patients, 26 (20.6\%) underwent a second pulmonary metastasectomy for pulmonary recurrence after initial pulmonary metastasectomy. Univariate analysis of survival identified seven significant factors: (1) gender $(p=0.04)$, (2) past history of extrathoracic metastasis $(p=0.04)$, (3) maximum tumor size $(p=0.002)$, (4) mediastinal lymph node metastasis $(p=0.02)$, (5) preoperative carcinoembryonic antigen (CEA) level $(p=0.01)$, (6) preoperative carbohydrate antigen 19-9 (CA199) level $(p=0.03)$, and (7) repeat pulmonary metastasectomy for pulmonary recurrence $(p<0.001)$. On multivariate analysis, only mediastinal lymph node metastasis $(p=0.02$, risk ratio $8.206,95 \%$ confidence interval $(\mathrm{Cl}) 1.566-$ $34.962)$ and repeat pulmonary metastasectomy for pulmonary recurrence $(p<0.001$, risk ratio $0.054,95 \% \mathrm{Cl} 0.010-$ 0.202) were significant. Furthermore, in the evaluation of surgical outcomes, the safety of second pulmonary metastasectomy was almost the same as that of initial pulmonary metastasectomy.

Conclusions: Repeat pulmonary metastasectomy is likely to be safe and effective for recurrent cases that meet the surgical criteria. However, mediastinal lymph node metastasis was a significant independent prognostic factor for worse overall survival.
\end{abstract}

Keywords: Colorectal cancer, Pulmonary metastasis, Repeat pulmonary metastasectomy, Prognostic factor, Mediastinal lymph node metastasis

\footnotetext{
* Correspondence: kyoshida@gifu-u.ac.jp

'Department of Surgical Oncology, Graduate School of Medicine, Gifu University, 1-1 Yanagido, Gifu City, Gifu 501-1194, Japan

Full list of author information is available at the end of the article
}

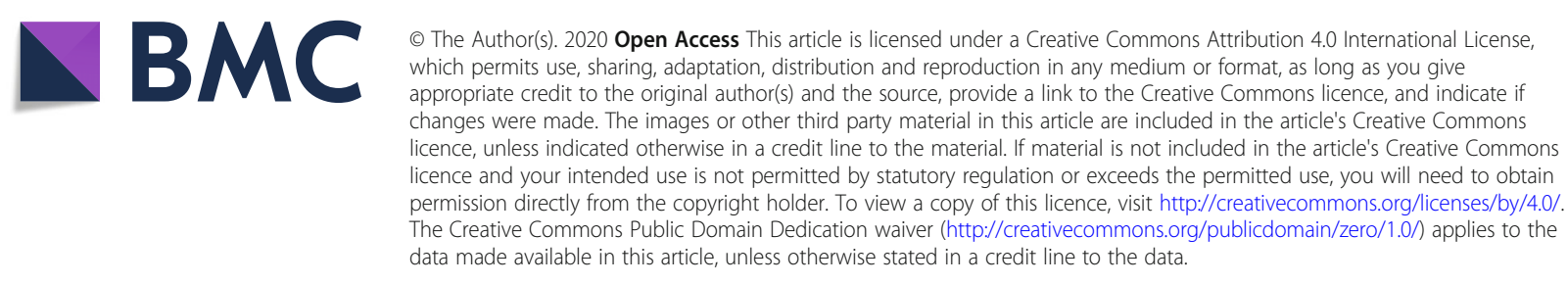




\section{Introduction}

Colorectal cancer (CRC) is one of the most common cancers and is known to metastasize frequently to the liver and lungs via the systemic blood flow. In the past, pulmonary metastasis was considered to be an indicator of cancer spread throughout the body, and aggressive treatment was commonly avoided. However, because of recent advances in chemotherapy, diagnostic techniques, and surgical procedures, pulmonary resection is widely accepted as the optimal treatment for pulmonary metastases [1-8]. It is expected that the clinical results of pulmonary metastasectomy will change with the improvement of medical care. Thus, it is necessary to continue evaluation of the outcome of pulmonary metastasectomy in CRC patients in order to identify true prognostic factors and determine appropriate surgical criteria. We report the recent clinical outcomes of pulmonary metastasectomy at our institutes. The main purpose of this study was to answer the following questions: (1) What are the potential prognostic factors for patients undergoing pulmonary metastasectomy? (2) What is the role of repeat pulmonary metastasectomy for recurrent metastatic CRC?

\section{Patients and methods}

\section{Study population}

A total of 126 patients underwent pulmonary metastasectomy at the Department of Thoracic Surgery, Gifu University Hospital, between March 2000 and December 2019. The study's retrospective protocol was approved by our institutional review board (approval number '2019-253'). Among the 126 patients included, 47 (37.3\%) had pulmonary recurrence after initial pulmonary metastasectomy, and $26(20.6 \%)$ who met the surgical criteria underwent a second pulmonary metastasectomy (Fig. 1).

All patients who underwent pulmonary metastasectomy met the following criteria based on the Japanese Society for Cancer of the Colon and Rectum (JSCCR) Guidelines for the treatment of CRC [9]: (1) the patient was capable of tolerating surgery; (2) the primary colorectal tumor was controlled or could be controlled; (3) the metastatic lung tumor could be completely resected; (4) any extra-thoracic metastases could be controlled; and (5) the function of the remaining lung would be adequate.

A controllable tumor is a tumor that can be completely resected, or a tumor without the appearance of

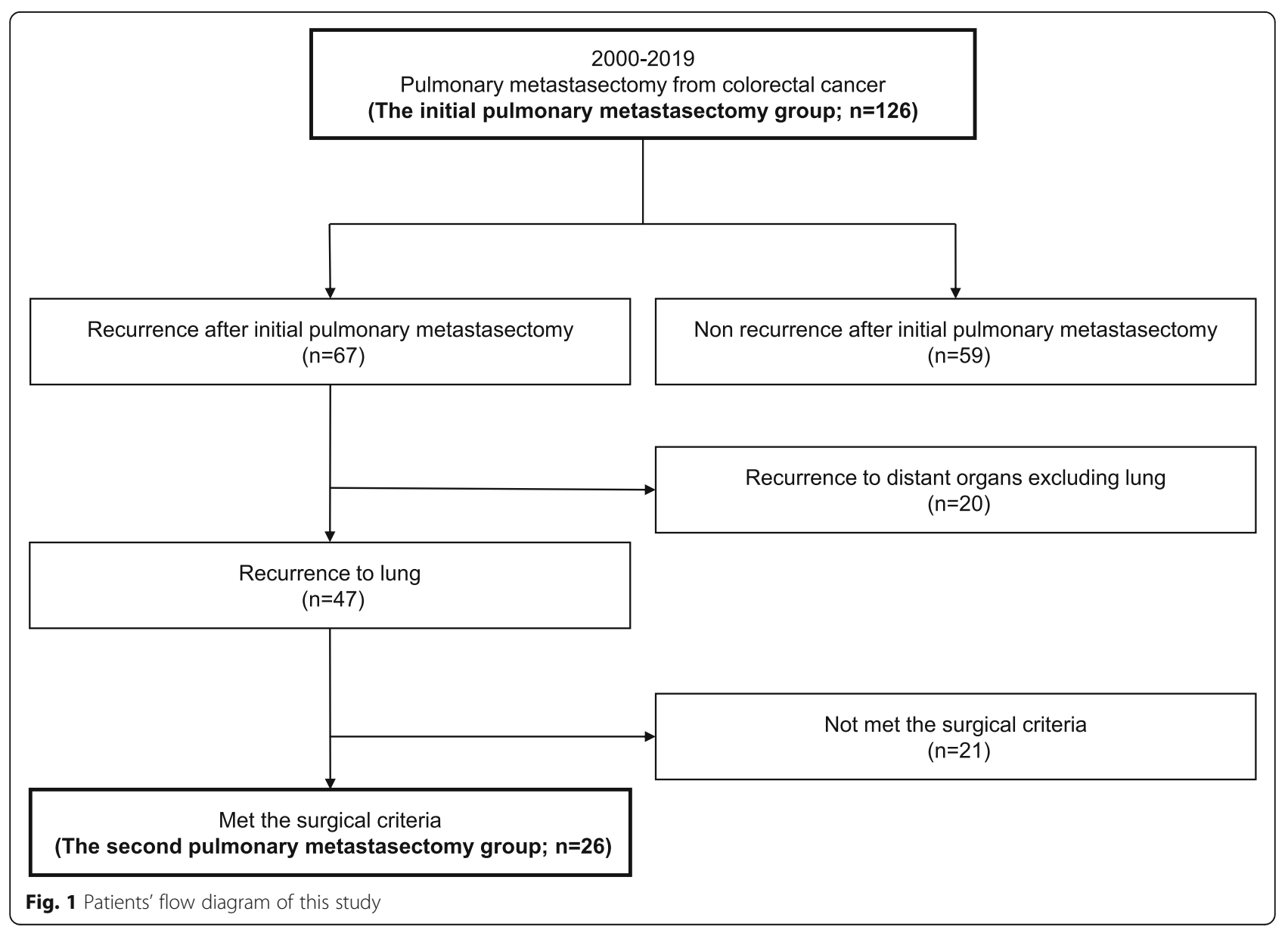


new lesions and regrowth after treatment such as surgery, chemotherapy, and radiation.

Preoperative assessments included clinical examination, blood tests, electrocardiogram, standard chest radiograph, spirometry, echocardiogram, contrastenhanced computed tomography scan (CT) of the chest and abdomen, and positron emission tomography (PET) whole body scan. Endobronchial ultrasound-guided transbronchial biopsy was not performed routinely in this study.

Regarding the extent of pulmonary resection, if metastatic tumor was anatomically present in the outer third of the lung and partial resection was possible, we performed partial resection. If it was located inside the lung, segmentectomy and lobectomy were performed.

Mediastinal lymph node dissection was performed according to primary lung cancer in the cases in which lymph node metastasis was suspected (swelling with short axis on CT $\geqq 10 \mathrm{~mm}$ and PET-positive). If lymph node metastasis was suspected during surgery, this lymph node was submitted to intraoperative consultation. If positive, mediastinal lymph node dissection was performed. In addition, in the case of lobectomy and segmentectomy, the regional and interlobar lymph nodes were dissected. Otherwise, mediastinal lymph node dissection was not performed.

The surgery was considered curative if all known pulmonary nodules were removed. Patients who had complete resection of all known pulmonary disease were included in this study. We reviewed each patient's medical records to obtain clinicopathological information of the initial and second pulmonary metastasectomy.

We collected information on patients and primary colorectal tumor characteristics including gender, age at the initial pulmonary metastasectomy, smoking habits (non-smoker or smoker), Brinkman index, primary colorectal tumor location (colon or rectum/right or left side), histological differentiation of the primary colorectal tumor (well, moderately, or poorly differentiated), pathological Union for International Cancer Control-TNM classification (8th edition) [10] of the primary colorectal tumor, past history of extra-thoracic metastasis (present or absent), adjuvant chemotherapy after the primary colorectal operation (yes or no), and the number of pulmonary metastasectomies.

The clinical characteristics of pulmonary metastases included diagnosis period (synchronous or metachronous), number (solitary or multiple), location (unilateral or bilateral), disease-free interval, maximum tumor size, mediastinal lymph node metastasis (positive or negative in postoperative histological lymph node status), preoperative carcinoembryonic antigen (CEA) (normal or elevated, normal upper limit being $5 \mathrm{ng} / \mathrm{ml}$ ), preoperative carbohydrate antigen 19-9 (CA19-9) (normal or elevated, normal upper limit being $37 \mathrm{ng} / \mathrm{ml}$ ), perioperative chemotherapy (yes or no), recurrence after pulmonary metastasectomy (yes or no), and recurrent distant organ. In this study, lung lesions diagnosed within 1 year from resection of the primary colorectal tumor were defined as synchronous metastases, and those diagnosed after 1 year were defined as metachronous metastases. The disease-free interval (DFI) referred to both the period from primary colorectal tumor resection to diagnosis of the initial pulmonary metastasis and the period from the initial pulmonary metastasectomy to diagnosis of the second pulmonary metastasis. In our department, as a general rule, perioperative chemotherapy was indicated in cases excluding solitary pulmonary metastasis with DFI $>1$ year.

Finally, the surgical characteristics of pulmonary metastasectomy were operation method (partial resection, segmentectomy, lobectomy/video-assisted thoracic surgery (VATS), or open surgery), operation time, intraoperative blood loss, preoperative percent vital capacity (\%VC), preoperative forced expiratory volume percent in $1 \mathrm{~s}\left(\mathrm{FEV}_{1.0} \%\right)$, preoperative respiratory dysfunction (absent or present), postoperative complications after pulmonary metastasectomy (Clavien-Dindo classification [11] grade $\geq 2$ : yes or no), postoperative mortality, and hospital stay.

\section{Statistical analysis}

For comparisons of variables between the initial and second pulmonary metastasectomy groups, Fisher's exact test was used for categorical variables, and the MannWhitney $U$ test was used for continuous and ordinal variables.

Overall survival was calculated in months from the date of the initial pulmonary resection to the date of the last follow-up. All cumulative survival curves were estimated using the Kaplan-Meier method, and in the univariate analysis, the log-rank test was used to evaluate differences between groups. A Cox relative risk regression model was used to estimate risk ratios and 95\% confidence intervals (CIs) for multivariate analysis. The significance level was set at $<0.05$. All statistical analyses were performed using JMP software (SAS Institute Inc., Cary, NC, USA).

\section{Results}

\section{Patient demographics}

Patient and primary colorectal tumor characteristics for each group are presented in Table 1 . The cohort consisted of 85 males (67.5\%) and 41 females (32.5\%). The age at the initial pulmonary metastasectomy ranged from 37 to 84 years, with a median of 66 years. The primary tumor location was the colon in 59 cases (46.8\%) and the rectum in 66 cases (52.4\%). Forty-five patients 
Table 1 Patients and primary colorectal tumor characteristics in each group (initial and second pulmonary metastasectomy)

\begin{tabular}{|c|c|c|c|}
\hline Characteristics & $\begin{array}{l}\text { The initial pulmonary } \\
\text { metastasectomy } \\
n=126\end{array}$ & $\begin{array}{l}\text { The second pulmonary } \\
\text { metastasectomy } \\
n=26\end{array}$ & $p$ value \\
\hline Gender, $n$ (\%) & $\begin{array}{l}\text { Male } 85(67.5) \\
\text { Female } 41(32.5)\end{array}$ & $\begin{array}{l}\text { Male } 16(61.5) \\
\text { Female } 10(38.5)\end{array}$ & 0.56 \\
\hline Age $\mathbb{1}$, median [range] & $66[37-84]$ & $62.5[37-77]$ & $0.04^{*}$ \\
\hline Smoking habits, $n$ (\%) & $\begin{array}{l}\text { Non-smoker } 39 \text { (31.0) } \\
\text { Smoker } 79(62.7)\end{array}$ & $\begin{array}{l}\text { Non-smoker } 10(38.5) \\
\text { Smoker } 13(50.0)\end{array}$ & 0.34 \\
\hline Brinkman indext, median [range] & $457.5[0-2100]$ & $107[0-2000]$ & 0.46 \\
\hline \multirow[t]{2}{*}{ Primary colorectal tumor location, $n(\%)$} & $\begin{array}{l}\text { Colon } 59(46.8) \\
\text { Rectum } 66(52.4)\end{array}$ & $\begin{array}{l}\text { Colon } 11(42.3) \\
\text { Rectum } 16(57.7)\end{array}$ & 0.65 \\
\hline & $\begin{array}{l}\text { Right side } 23(18.2) \\
\text { Left side } 102(81.0)\end{array}$ & $\begin{array}{l}\text { Right side } 3(11.5) \\
\text { Left side } 23(88.5)\end{array}$ & 0.38 \\
\hline $\begin{array}{l}\text { Histological differentiation of the } \\
\text { primary colorectal tumor, } n(\%)\end{array}$ & $\begin{array}{l}\text { Well-55 (43.6) } \\
\text { Moderately-53 (42.0) } \\
\text { Poorly-6 (4.8) }\end{array}$ & $\begin{array}{l}\text { Well-8 (30.8) } \\
\text { Moderately-9 (34.6) } \\
\text { Poorly-1 (3.8) }\end{array}$ & 0.54 \\
\hline Pathological T stage $\neq, n(\%)$ & $\begin{array}{l}\text { T1 } 7 \text { ( } 5.6) \\
\text { T2 } 11(8.7) \\
\text { T3 } 61(48.4) \\
\text { T4 } 37(29.4)\end{array}$ & $\begin{array}{l}\text { T1 } 1 \text { ( (3.8) } \\
\text { T2 } 0(0.0) \\
\text { T3 } 15(57.7) \\
\text { T4 } 6(23.1)\end{array}$ & 0.19 \\
\hline Pathological N stage $\neq, n(\%)$ & $\begin{array}{l}\text { No } 43(34.1) \\
\text { N1 } 47(37.3) \\
\text { N2 } 24(19.0)\end{array}$ & $\begin{array}{l}\text { N0 } 7(27.0) \\
\text { N1 } 9(34.6) \\
\text { N2 } 5(19.2)\end{array}$ & 0.92 \\
\hline Pathological stage $\neq, n(\%)$ & $\begin{array}{l}\text { I } 8(6.3) \\
\text { II } 25(19.8) \\
\text { III } 56(44.4) \\
\text { IV } 31(24.6)\end{array}$ & $\begin{array}{l}\text { I } 0(0.0) \\
\text { II } 5(19.2) \\
\text { III } 12(46.2) \\
\text { IV } 8(30.8)\end{array}$ & 0.35 \\
\hline $\begin{array}{l}\text { Past history of extra thoracic metastasis, } n \\
\text { (\%) }\end{array}$ & $\begin{array}{l}\text { Present } 45(35.7) \\
\text { Absent } 81(64.3)\end{array}$ & $\begin{array}{l}\text { Present } 10(38.5) \\
\text { Absent } 16(61.5)\end{array}$ & 0.79 \\
\hline Adjuvant chemotherapy, $n$ (\%) & Yes 61 (48.4) No 57 (45.2) & Yes 16 (61.5) No 8 (30.8) & 0.18 \\
\hline Regimen, $n(\%)$ & $\begin{array}{l}\text { UFT } 28 \text { (22.2) capecitabine } 7 \text { (5.6) CapeOX } 7 \text { (5.6) FOLFOX } 5 \\
\text { (4.0) } \\
\text { S1 } 4 \text { (3.2) FU + LV } 3 \text { (2.4) } \\
\text { Other } 7 \text { (5.6) }\end{array}$ & $\begin{array}{l}\text { UFT } 5 \text { (19.2) FOLFOX } 3 \text { (11.5) } \\
\text { Capecitabine } 2 \text { (7.7) CapeOX } 1 \\
(3.8) \\
\text { FU + LV } 1 \text { (3.8) } \\
\text { Other } 4 \text { (15.4) }\end{array}$ & - \\
\hline $\begin{array}{l}\text { Number of pulmonary metastasectomy, } n \\
(\%)\end{array}$ & - & $\begin{array}{l}2 \text { times } 14(53.9) \\
3 \text { times } 7(26.9) \\
4 \text { times } 5(19.2)\end{array}$ & - \\
\hline
\end{tabular}

ף: Age at the initial pulmonary metastasectomy

$\mathbf{t}$ : Brinkman index $=$ (the number of cigarette smoked per day) $\times$ (the number of years of smoking)

\#: UICC TNM classification(the $8^{\text {th }}$ edition)

${ }^{*} p<0.05 ;{ }^{* *} p<0.01 ;{ }^{* * *} p<0.001$

(35.7\%) had a past history of extra-thoracic metastasis, and the liver (33 cases, 26.2\%) was the most frequent site of metastasis.

In the second pulmonary metastasectomy group, the mode and maximum number of repeat pulmonary metastasectomies were 2 (14 cases, 53.9\%) and 4 (5 cases, $19.2 \%)$, respectively. The age at the initial metastasectomy was significantly younger $(p=0.04)$. However, there was no significant difference in other patient and primary colorectal tumor characteristics between the two groups.

The characteristics of pulmonary metastases in each group are presented in Table 2 . There was no significant difference between the two groups in 10 clinical characteristics. Although the DFI was not significantly different between the groups (median 541.5 (range 04664) days vs 409 (range 27-1334) days, $p=0.13\}$, the recurrence rate tended to be higher in the second pulmonary resection group $(53.2 \%$ vs $65.4 \%, p=0.07)$. The lung was the most common metastatic organ in both groups (37.3\% and $46.4 \%$, respectively).

\section{Surgical characteristics in initial and second pulmonary metastasectomy}

Surgical characteristics for each group are presented in Table 3. The amount of intraoperative blood loss was significantly higher in patients undergoing second pulmonary metastasectomy \{median 10 (range 0-1130) ml 
Table 2 Pulmonary metastases characteristics in each group (initial and second pulmonary metastasectomy)

\begin{tabular}{|c|c|c|c|c|}
\hline \multicolumn{2}{|l|}{ Characteristics } & $\begin{array}{l}\text { The initial pulmonary } \\
\text { metastasectomy } \\
n=126\end{array}$ & $\begin{array}{l}\text { The second pulmonary } \\
\text { metastasectomy } \\
n=26\end{array}$ & $p$ value \\
\hline \multicolumn{2}{|l|}{ Diagnosis period, $n(\%)$} & $\begin{array}{l}\text { Synchronous } 41 \text { (32.5) } \\
\text { Metachronous } 85 \text { (67.5) }\end{array}$ & - & - \\
\hline \multicolumn{2}{|l|}{ Number of pulmonary metastasis, $n(\%)$} & $\begin{array}{l}1: 89(70.6) \\
2: 17(13.5) \\
3: 12(9.5) \\
4: 3(2.4) \\
5: 2(1.6)\end{array}$ & $\begin{array}{l}\text { 1: } 16 \text { (61.5) } \\
\text { 2: } 7(26.9) \\
\text { 3: } 1(3.8) \\
4: 2(7.7)\end{array}$ & 0.36 \\
\hline & Solitary & 89 (70.6) & $16(61.5)$ & \multirow[t]{2}{*}{0.27} \\
\hline & Multiple & $34(27.0)$ & $10(38.5)$ & \\
\hline \multirow[t]{2}{*}{ Location, $n(\%)$} & Unilateral & $108(85.7)$ & $23(88.5)$ & \multirow[t]{2}{*}{0.71} \\
\hline & Bilateral & $18(14.3)$ & $3(11.5)$ & \\
\hline \multicolumn{2}{|c|}{ Disease free interval (days), median [range] } & $541.5[0-4664]$ & 409 [27-1334] & 0.13 \\
\hline \multicolumn{2}{|l|}{ Maximum tumor size (mm), median [range] } & $12.0[5-70]$ & $12.0[8-40]$ & 1.00 \\
\hline \multirow{2}{*}{$\begin{array}{l}\text { Mediastinal lymph node metastasis, } \\
n(\%)\end{array}$} & Negative & $117(92.9)$ & $25(96.2)$ & \multirow[t]{2}{*}{0.95} \\
\hline & Positive & $5(4.0)$ & $1(3.8)$ & \\
\hline \multirow[t]{2}{*}{ Preoperative CEA level, $n$ (\%) } & Normal & $86(68.3)$ & $15(57.7)$ & \multirow[t]{2}{*}{0.61} \\
\hline & Elevated & $36(28.6)$ & $8(30.8)$ & \\
\hline \multirow[t]{2}{*}{ Preoperative CA19-9 level, $n$ (\%) } & Normal & $97(77.0)$ & $21(80.8)$ & \multirow[t]{2}{*}{0.74} \\
\hline & Elevated & $12(9.5)$ & $2(7.7)$ & \\
\hline \multirow[t]{2}{*}{ Chemotherapy before operation, $n(\%)$} & Yes & $28(22.2)$ & $9(34.6)$ & \multirow[t]{2}{*}{0.09} \\
\hline & No & $96(76.2)$ & $15(57.7)$ & \\
\hline \multirow[t]{2}{*}{ Chemotherapy after operation, $n$ (\%) } & Yes & $40(31.7)$ & $9(34.6)$ & \multirow[t]{2}{*}{0.50} \\
\hline & No & $74(58.7)$ & $12(46.2)$ & \\
\hline \multirow{2}{*}{$\begin{array}{l}\text { Recurrence after pulmonary } \\
\text { metastasectomy, } n(\%)\end{array}$} & Yes & $67(53.2)$ & $17(65.4)$ & \multirow[t]{2}{*}{0.07} \\
\hline & No & $59(46.8)$ & $7(26.9)$ & \\
\hline \multicolumn{2}{|l|}{ Recurrent organ, $n(\%)$} & $\begin{array}{l}\text { Lung } 47(37.3) \\
\text { Liver } 12 \text { (9.5) } \\
\text { Abdominal lymph node } 6(4.8) \\
\text { Pelvic local recurrence } 6(4.8) \\
\text { Bone } 5 \text { (4.0) Brain } 5(4.0) \\
\text { Peritoneal dissemination } 3(2.4) \\
\text { Pleural dissemination } 2(1.6) \\
\text { Bone marrow } 1 \text { (0.8) Adrenal } 1(0.8)\end{array}$ & \multicolumn{2}{|l|}{$\begin{array}{l}\text { Lung } 13 \text { (46.4) } \\
\text { Pleural dissemination } 2 \text { (7.2) } \\
\text { Thoracic lymph node } 2 \text { (7.2) } \\
\text { Liver } 1 \text { (3.6) Bone } 1 \text { (3.6) } \\
\text { Brain } 1 \text { (3.6) Pancreas } 1 \text { (3.6) }\end{array}$} \\
\hline
\end{tabular}

CEA carcinoembryonic antigen level (normal upper limit at $5 \mathrm{ng} / \mathrm{ml}$ ), CA19-9 carbohydrate antigen 19-9 level (normal upper limit at $37 \mathrm{ng} / \mathrm{ml}$ ) ${ }^{*} p<0.05 ;{ }^{* *} p<0.01 ;{ }^{* * *} p<0.001$

vs $20(0-220) \mathrm{ml}, p=0.008\}$. However, there were no significant differences in postoperative complications, postoperative mortality, or hospital stay between the groups.

\section{Survival following the pulmonary metastasectomy from CRC}

The median follow-up period after the primary pulmonary metastasectomy was 37 months (range 1-209 months). Of the 126 patients, 33 (24.3\%) died after pulmonary metastasectomy: 24 (19.0\%) of CRC, 8 patients (6.3\%) of another disease, and $1(0.8 \%)$ of unknown causes.
The 3- and 5-year survival rates of all 126 patients who underwent complete pulmonary metastasectomy were $84.9 \%$ and $60.8 \%$, respectively (Fig. 2). Table 4 lists the 5-year survival rates after the pulmonary metastasectomy according to 22 clinicopathological features. Univariate analysis identified seven significant factors: (1) gender $(p=0.04),(2)$ past history of extra-thoracic metastasis $(p=0.04)$, (3) maximum tumor size $(p=$ $0.002)$, (4) mediastinal lymph node metastasis $(p=0.02)$, (5) preoperative CEA level $(p=0.01),(6)$ preoperative CA19-9 level $(p=0.03)$, and (7) repeat pulmonary metastasectomy for pulmonary recurrence $(p<0.001)$. Multivariate analysis using a Cox relative risk regression model indicated that of these features (Table 5), 
Table 3 Surgical characteristics in each group (initial and second pulmonary metastasectomy)

\begin{tabular}{|c|c|c|c|c|}
\hline Characteristics & & $\begin{array}{l}\text { The initial pulmonary } \\
\text { metastasectomy }(n=126)\end{array}$ & $\begin{array}{l}\text { The second pulmonary } \\
\text { metastasectomy } \\
(n=26)\end{array}$ & $p$ value \\
\hline \multirow[t]{5}{*}{ Operation, $n(\%)$} & Partial resection & $65(51.6)$ & $8(30.8)$ & \multirow[t]{3}{*}{0.09} \\
\hline & Segmentectomy & $31(24.6)$ & $11(42.3)$ & \\
\hline & Lobectomy & $30(23.8)$ & $5(19.2)$ & \\
\hline & VATS & $106(84.1)$ & $17(65.4)$ & \multirow[t]{2}{*}{0.12} \\
\hline & Open & $20(15.9)$ & $7(26.9)$ & \\
\hline Operation time (min), median [range] & & $163[40-645]$ & 217 [62-505] & 0.15 \\
\hline Intraoperative blood loss (ml), median [range] & & $10[0-1130]$ & $20[0-220]$ & $0.008^{* *}$ \\
\hline Preoperative \%VC (\%), median [range] & & $109.8[56.7-154]$ & $109.0[56.4-147]$ & 0.23 \\
\hline Preoperative $\mathrm{FEV}_{1.0} \%(\%)$, median [range] & & $74.9[46.8-102.6]$ & $72.7[59.8-97.1]$ & 0.99 \\
\hline \multirow[t]{5}{*}{ Preoperative respiratory dysfunction, $n$ (\%) } & Absent & $77(61.1)$ & $13(50.0)$ & \multirow[t]{5}{*}{0.91} \\
\hline & Present & $44(34.9)$ & $10(38.5)$ & \\
\hline & Restrictive & $4(3.2)$ & $2(7.7)$ & \\
\hline & Obstructive & $39(31.0)$ & $8(30.8)$ & \\
\hline & Mixed & $1(0.8)$ & $0(0.0)$ & \\
\hline \multirow{3}{*}{$\begin{array}{l}\text { Postoperative complication } \\
\left(\geqq C D^{\mathrm{a}} \text {-grade2), } n(\%)\right.\end{array}$} & Yes & $5(3.9)$ & $0(0.0)$ & \multirow[t]{3}{*}{0.32} \\
\hline & No & $115(91.3)$ & $24(92.3)$ & \\
\hline & & $\begin{array}{l}\text { Fistula } 2 \text { (1.6) } \\
\text { Pneumonia } 1(0.8) \\
\text { Air leakage } 1(0.8) \\
\text { Pleural effusion } 1 \text { (0.8) }\end{array}$ & - & \\
\hline Postoperative mortality, n (\%) & & $0(0.0)$ & $0(0.0)$ & - \\
\hline Hospital stay (day), median [range] & & $7[2-55]$ & 8 [3-19] & 0.51 \\
\hline
\end{tabular}

VATS Video-assisted thoracic surgery, \%VC percent vital capacity, FEV $V_{1.0} \%$ forced expiratory volume percent in $1 \mathrm{~s}$

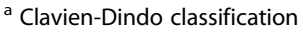

${ }^{*} p<0.05 ;{ }^{* *} p<0.01 ;{ }^{* * *} p<0.001$

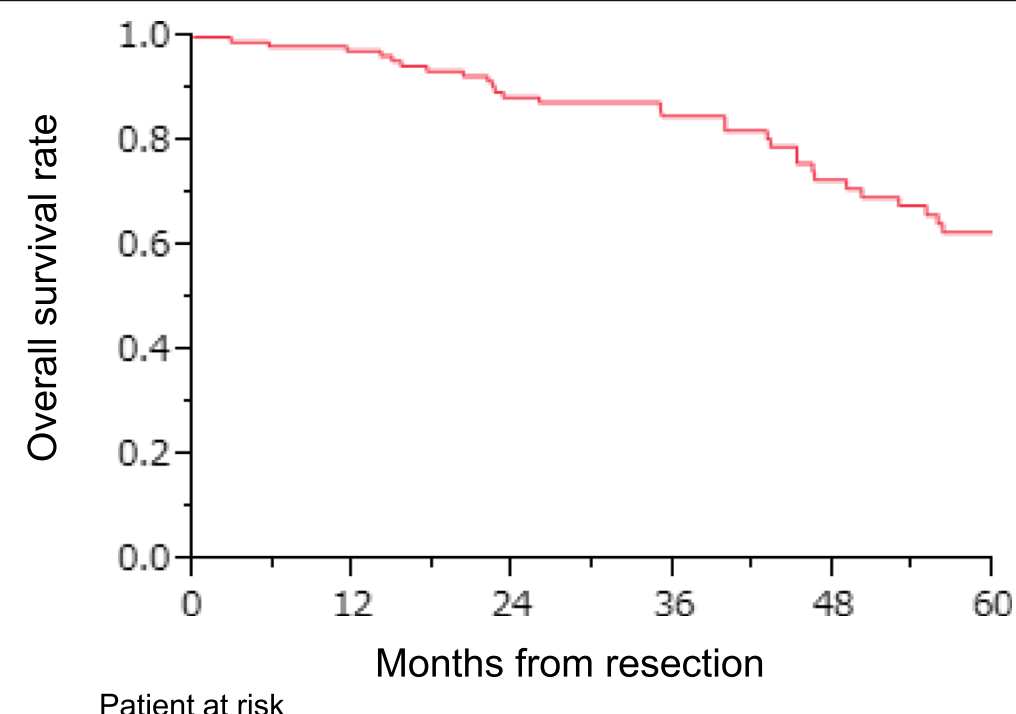

126

106

86

65

46

33

Fig. 2 Overall survival of pulmonary metastasectomy for colorectal cancer 
Table 4 Survival of the pulmonary metastasectomy from CRC in univariate analysis

\begin{tabular}{|c|c|c|c|c|}
\hline Prognostic factors & & $n(\%)$ & $\begin{array}{l}5 \text {-year overall survival after the initial } \\
\text { pulmonary metastasectomy (\%) }\end{array}$ & $p$ value \\
\hline \multirow[t]{2}{*}{ Gender } & Male & $85(67.5)$ & 54.5 & $0.04^{*}$ \\
\hline & Female & $41(32.5)$ & 77.0 & \\
\hline \multirow[t]{2}{*}{ Age (years) } & $\geqq 70$ & $41(32.5)$ & 53.8 & 0.34 \\
\hline & $<70$ & $85(67.5)$ & 63.1 & \\
\hline \multirow[t]{2}{*}{ Brinkman index ${ }^{\mathrm{a}}$} & $\geqq 400$ & $58(46.0)$ & 64.5 & 0.78 \\
\hline & $<400$ & $48(38.1)$ & 59.2 & \\
\hline \multirow[t]{4}{*}{ Primary colorectal tumor location } & Colon & $59(46.8)$ & 53.4 & 0.31 \\
\hline & Rectum & $66(52.4)$ & 69.1 & \\
\hline & Right-sided & $23(18.2)$ & 57.3 & 0.50 \\
\hline & Left-sided & $102(81.0)$ & 61.3 & \\
\hline \multirow{2}{*}{$\begin{array}{l}\text { Histological differentiation of the } \\
\text { primary colorectal tumor }\end{array}$} & Well & $55(43.6)$ & 67.3 & 0.26 \\
\hline & Moderately and poorly & $59(46.8)$ & 59.8 & \\
\hline \multirow[t]{2}{*}{ Pathological T stage ${ }^{\mathrm{b}}$} & $\mathrm{T} 4$ & $37(29.4)$ & 55.2 & 0.21 \\
\hline & $T<4$ & $79(62.7)$ & 63.9 & \\
\hline \multirow[t]{2}{*}{ Pathological N stage ${ }^{\mathrm{b}}$} & $N \geqq 1$ & $71(56.3)$ & 61.6 & 0.99 \\
\hline & No & $43(34.1)$ & 60.5 & \\
\hline \multirow[t]{2}{*}{ Past history of extra thoracic metastasis } & Presence & 45 (35.7) & 53.1 & $0.04^{*}$ \\
\hline & Absence & $81(64.3)$ & 65.4 & \\
\hline \multirow[t]{2}{*}{ Past history of liver metastasis } & Presence & $33(26.2)$ & 52.4 & 0.05 \\
\hline & Absence & $93(73.8)$ & 64.3 & \\
\hline \multirow{2}{*}{$\begin{array}{l}\text { Adjuvant chemotherapy after primary } \\
\text { colorectal resection }\end{array}$} & Yes & $61(48.4)$ & 68.5 & 0.07 \\
\hline & No & $57(45.2)$ & 53.9 & \\
\hline \multirow[t]{2}{*}{ Diagnosis period of pulmonary metastases } & Synchronous & $41(32.5)$ & 63.7 & 0.48 \\
\hline & Metachronous & $85(67.5)$ & 59.3 & \\
\hline \multirow[t]{2}{*}{ Number of pulmonary metastases } & Solitary & 89 (70.6) & 62.2 & 0.75 \\
\hline & Multiple & $34(27.0)$ & 55 & \\
\hline \multirow[t]{2}{*}{ Location of pulmonary metastases } & Unilateral & $108(85.7)$ & 62.9 & 0.56 \\
\hline & Bilateral & $18(14.3)$ & 52.9 & \\
\hline \multirow[t]{2}{*}{ Maximum tumor size (mm) } & $\geqq 20$ & $27(21.4)$ & 31.2 & $0.002^{* *}$ \\
\hline & $<20$ & $94(74.6)$ & 66.1 & \\
\hline \multirow[t]{2}{*}{ Mediastinal lymph node metastasis } & Positive & $5(4.0)$ & 20.0 & $0.02^{*}$ \\
\hline & Negative & $117(74.6)$ & 60.8 & \\
\hline \multirow[t]{2}{*}{ Preoperative CEA level } & Normal & $86(68.3)$ & 67.7 & $0.01^{*}$ \\
\hline & Elevated & $36(28.6)$ & 37.3 & \\
\hline \multirow[t]{2}{*}{ Preoperative CA19-9 level } & Normal & $97(77)$ & 64.1 & $0.03^{*}$ \\
\hline & Elevated & $12(9.5)$ & 31.8 & \\
\hline \multirow{2}{*}{$\begin{array}{l}\text { Disease free interval after primary } \\
\text { colorectal resection (years) }\end{array}$} & $\geqq 2$ & $81(64.3)$ & 59.9 & 0.91 \\
\hline & $<2$ & $45(35.7)$ & 62.4 & \\
\hline \multirow{2}{*}{$\begin{array}{l}\text { Chemotherapy before pulmonary } \\
\text { metastasectomy }\end{array}$} & Yes & $28(22.2)$ & 49.6 & 0.17 \\
\hline & No & $96(76.2)$ & 62.6 & \\
\hline \multirow{2}{*}{$\begin{array}{l}\text { Chemotherapy after pulmonary } \\
\text { metastasectomy }\end{array}$} & Yes & $40(31.7)$ & 60.6 & 0.77 \\
\hline & No & $74(58.7)$ & 59.7 & \\
\hline \multirow{2}{*}{$\begin{array}{l}\text { Repeat pulmonary metastasectomy } \\
\text { for pulmonary recurrence }\end{array}$} & Yes & $26(38.8)$ & 76.9 & $<0.001^{* * *}$ \\
\hline & No & $41(61.2)$ & 8.7 & \\
\hline
\end{tabular}

CRC colorectal cancer, CEA carcinoembryonic antigen level, normal upper limit at $5 \mathrm{ng} / \mathrm{ml}$, CA19-9 carbohydrate antigen 19-9 level, normal upper limit at $37 \mathrm{ng} / \mathrm{ml}$

${ }^{\mathrm{a} B r i n k m a n}$ index $=$ (the number of cigarette smoked per day) $\times$ (the number of years of smoking)

bUICC TNM classification (the 8th edition)

${ }^{*} p<0.05 ;{ }^{* *} p<0.01 ;{ }^{* * *} p<0.001$ 
Table 5 Survival of the pulmonary metastasectomy from CRC in multivariate analysis

\begin{tabular}{llll}
\hline Prognostic factors & $\boldsymbol{p}$ value & Risk ratio & 95\% confidence interval \\
\hline Gender (male/female) & 0.60 & 1.324 & $0.484-4.381$ \\
Past history of extra thoracic metastasis (presence/absence) & 0.67 & 1.205 & $0.511-2.922$ \\
Preoperative CEA level (elevated/normal) & 0.89 & 1.083 & $0.356-3.547$ \\
Maximum tumor size ( $\geqq 20 \mathrm{~mm} /<20 \mathrm{~mm})$ & 0.74 & 1.203 & $0.401-3.646$ \\
Mediastinal lymph node metastasis (positive/negative) & $0.02^{*}$ & 8.206 & $1.566-34.962$ \\
Repeat pulmonary metastasectomy for the pulmonary recurrence (yes/no) & $<0.001^{* * *}$ & 0.054 & $0.010-0.202$
\end{tabular}

CRC colorectal cancer, CEA carcinoembryonic antigen level, normal upper limit at $5 \mathrm{ng} / \mathrm{ml}$ ${ }^{*} p<0.05 ;{ }^{* *} p<0.01 ;{ }^{* * * *} p<0.001$

preoperative CA19-9 level was excluded to avoid confounding with preoperative CEA level, and only mediastinal lymph node metastasis $(p=0.02$, risk ratio 8.206 , 95\% CI 1.566-34.962, Fig. 3) and repeat pulmonary metastasectomy for pulmonary recurrence $(p<0.001$, risk ratio $0.054,95 \%$ CI $0.010-0.202$, Fig. 4) were significant.

\section{Discussion}

The number of new CRC cases has been increasing annually worldwide. In 2002, the number of new diagnoses was estimated to be about 1.02 million globally [12], but in 2018, the number had increased to about 1.8 million [13]. Accordingly, the number of patients with pulmonary metastases from CRC is inevitably increasing. However, the development of multidrug chemotherapy regimens such as FOLFOX and FOLFIRI and the emergence of molecular targeting drugs such as antiVEGF antibody and anti-EGFR antibodies have dramatically improved CRC outcomes. Treatment strategies for pulmonary metastasis of CRC have received attention for the purpose of further improving prognosis [1-8].

Previously, pulmonary metastasis was considered to be a condition in which cancer spread throughout the body, and aggressive treatment was commonly avoided. However, since Thomfold et al. [14] proposed the principles of surgical treatment for pulmonary metastases, pulmonary metastasectomy has been performed on patients who meet the operative criteria, and the prognosis after treatment is relatively good. The 5 -year survival rate after pulmonary resection is reported to be $30-68 \%$ [1-8]; a similar result was observed in this study $(60.8 \%)$. In the multicenter aggregate in the JSCCR project study [8], the 5-year survival rate of lung resection cases was

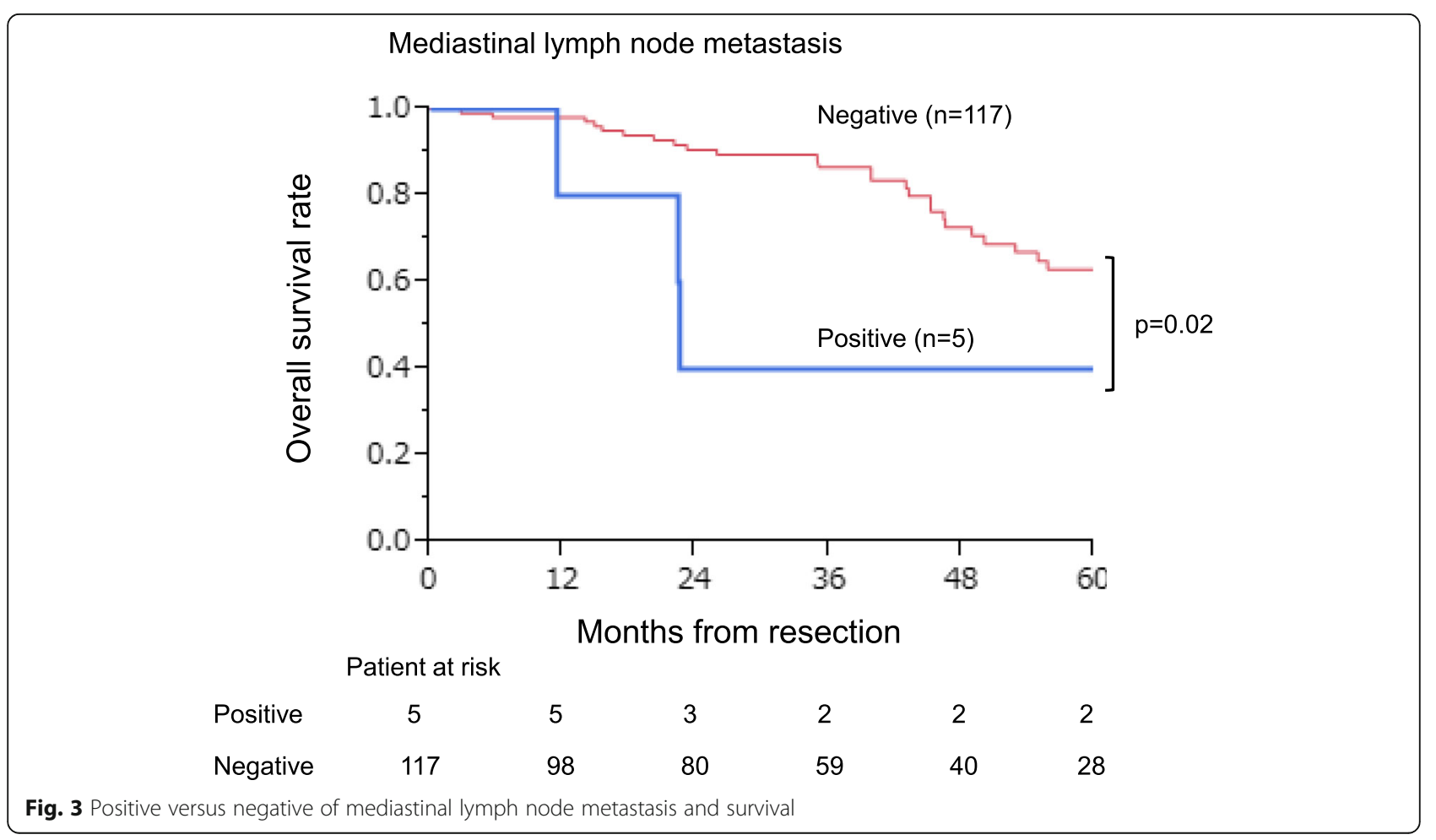




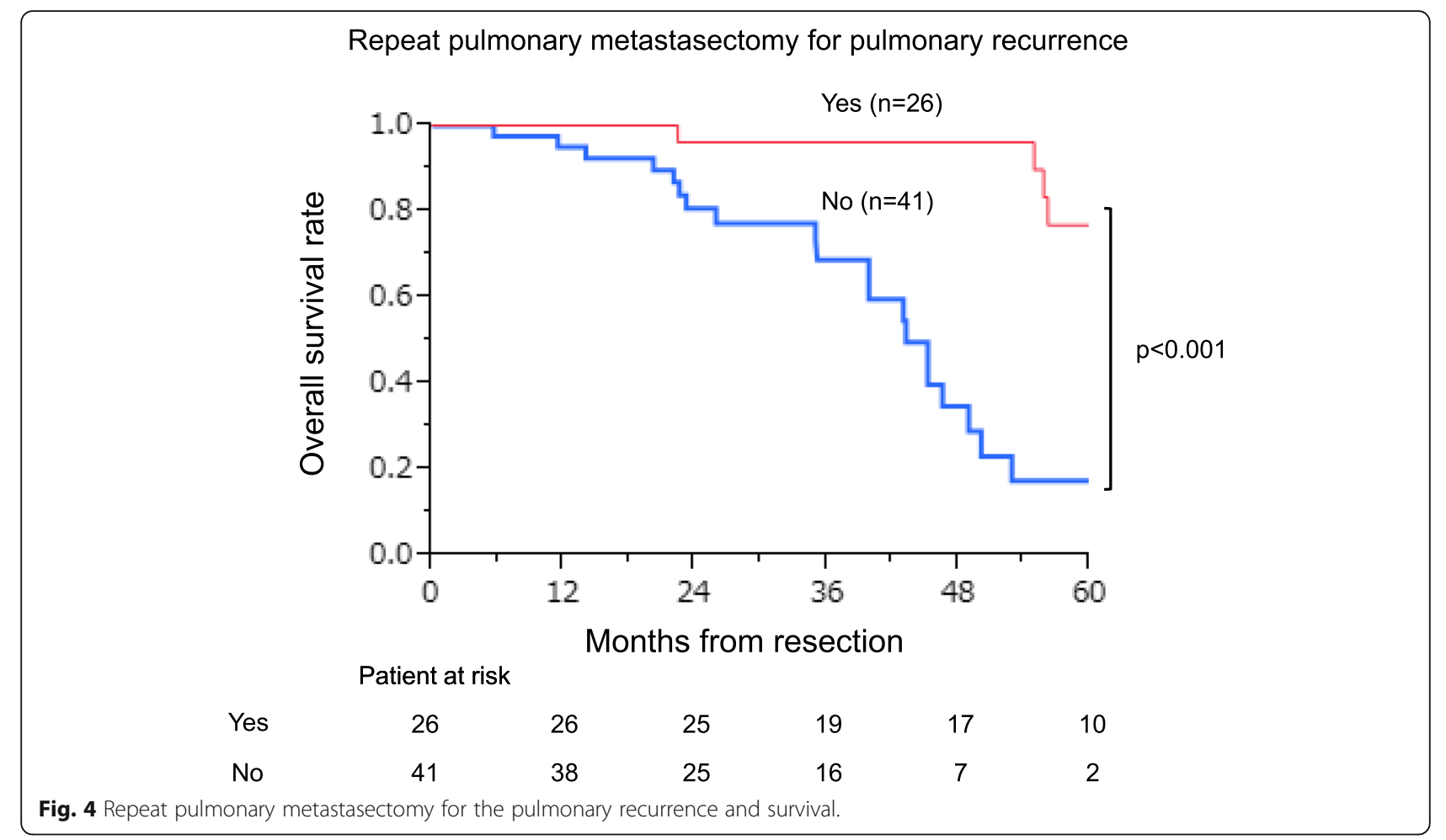

$46.7 \%$ and the cumulative 5-year relapse-free survival rate was $33.7 \%$, whereas the 5 -year survival rate of nonresected cases was $3.9 \%$. While many previous reports have shown good results in curative resection for lung metastases from CRC, the PULMICC (Pulmonary Metastasectomy in Colorectal Cancer) trial showed contrasting results [15]. This was a 2-armed multicenter randomized trial which compared pulmonary metastasectomy with continued observation for enrolling 65 patients. As a result, a 4-year overall survival was $40 \%$ in the control group versus $43 \%$ for patients assigned to metastasectomy. Although this trial was not able to demonstrate a survival benefit of metastasectomy, its outcome may be explained by the small sample size and the good prognosis of the control group which contrasts with the widely held assumption that patients with lung metastases have a 5 -year overall survival of $<5 \%[8,16]$. Thus, it is important to continue to accumulate cohort studies or randomized controlled trials of adequate sample size to reveal the true value of pulmonary metastasectomy.

According to some reports [1-8, 17-27], the number of metastases, location of lung metastases, mediastinal lymph node metastasis, CEA before pulmonary metastasectomy, primary colorectal tumor factors ( $\mathrm{T}$ factor and $\mathrm{N}$ factor), and DFI after resection of the primary colorectal tumor were found to be prognostic factors. In this study, history of extra-thoracic metastasis, maximum tumor size, mediastinal lymph node metastasis, and elevated tumor marker level before pulmonary metastasectomy were also identified as poor prognostic factors. Multivariate analysis identified only mediastinal lymph node metastasis as an independent predictor of poor prognosis. Therefore, excluding cases of mediastinal lymph node metastasis, our results suggest that neither the characteristics of the patients and the primary colorectal tumor nor those of the pulmonary metastases affected the prognosis after pulmonary metastasectomy.

Mediastinal lymph node metastasis in patients with pulmonary metastases is considered to reflect the spread of the cancer to the entire body and is therefore likely to be a poor prognostic factor. In our study, although the number of mediastinal lymph node-positive cases was small, all had past histories of extra-thoracic metastasis. Furthermore, distant metastases to other extra-thoracic organs such as the brain, liver, and bone occurred within 1 year after surgery in these patients. Several studies have suggested an association of mediastinal lymph node metastasis with an increased risk of death $[17,19,20,23$, 26-28], and a meta-analysis [22] showed poor 5-year survival among patients with lymph node metastasis (range, 0 to $33.5 \%$ ) compared to those without lymph node metastasis (range, 38.7 to $71 \%$ ). Our results suggest that lymph node dissection for patients with mediastinal lymph node metastasis has low therapeutic efficacy for those with other poor prognostic factors, and preventive systematic thoracic lymph node dissection to prolong prognosis is probably not necessary. Hamaji et al. 
reported that systematic lymph node dissection was not a significant factor for prolonged survival in the patients who underwent lymph node dissection, although longterm survivors were present [28]. Furthermore, Welter et al. [29] suggested it is more important to offer adjuvant chemotherapy after metastasectomy in cases of nodal metastasis than to perform radical or systematic lymph node dissection in patients with stage IV disease, bearing in mind the risk of recurrence in extrapulmonary organs. However, it was reported that CTand PET-based imaging levels have poor sensitivity (only $35 \%$ ) for detecting mediastinal lymph node metastasis [28]. In this study as well, the sensitivity of preoperative examination for mediastinal lymph node was $60 \%$, despite a good specificity. Therefore, thoracic lymph node dissection may have clinical significance as a diagnostic tool for prognostic purpose and a direction of the decision towards an adjuvant systemic chemotherapy. In our study, perioperative chemotherapy had no significant effect on survival. Prospective studies on the efficacy of perioperative chemotherapy and appropriate indications for it are necessary in the future.

The present study also showed that repeat pulmonary metastasectomy for pulmonary recurrence is likely to be effective. Repeat pulmonary metastasectomy is a wellestablished procedure with satisfactory survival $[1,2,4$, $5,17,18,21]$. We had 26 patients $(20.6 \%$ of all 126 cases and $55.3 \%$ of the 47 pulmonary recurrent cases after initial pulmonary metastasectomy) who underwent repeat pulmonary metastasectomy. They had 1- and 3-year survivals of $90.7 \%$ and $84.6 \%$, respectively, after the second pulmonary metastasectomy, which are similar to the outcomes after initial metastasectomy $(97.4 \%$ and $84.9 \%$, respectively). There was no significant difference between the two groups in either the clinical characteristics of the primary colorectal tumor and the pulmonary metastases, or in the surgical outcomes including postoperative complications, mortality, and length of hospital stay. Only the amount of intraoperative blood loss was significantly higher in the second pulmonary metastasectomy group, probably because of the higher rate of segmentectomy. Furthermore, the difference in the amount of blood loss was so small that it was considered of no clinical significance. Therefore, at least one repeat pulmonary metastasectomy can be performed relatively safely and can be expected to improve the prognosis if strictly complying with operative criteria.

As a secondary analysis, the prognostic factors for the second pulmonary metastasectomy group were also evaluated by the same method. In past reports [21, 30-32], preoperative CEA level, number of pulmonary metastases, mediastinal lymph node metastasis, and DFI were found to affect survival after repeat pulmonary metastasectomy. In this study, it was difficult to draw a firm conclusion due to sample size and no prognostic factors showing significant differences were found in the patients who met the surgical criteria.

Some limitations of this study have to be addressed. First, the major limitation of our study is the singleinstitution, retrospective design. Second, there was a potential for selection bias, which was compounded by the retrospective design. Inclusion of patients was highly selective, with patients having presumed good performance status and few comorbidities, which might have contributed to the observed long-term survival. In this study, complete resection of lung lesions is one of the important surgical criteria. Therefore, the surgeon's aggressiveness, experience, and skill probably have an impact. As a result, some features of pulmonary metastases, such as the number of metastases reported in the past as prognostic factors, may not show significant differences. These limitations should be considered when evaluating the results of the present study. It is necessary to carry out a prospective study with an appropriate control group at multiple institutions that have a unified definition of operative indication and treatment strategy. The medical treatment of malignant tumor has entered the era of immunotherapy [33]. Compared with traditional chemotherapy, neoadjuvant and adjuvant immunotherapy may improve the survival rate of patients. Thus, the study of treatment including immunotherapy will be needed in the future.

\section{Conclusions}

Pulmonary metastasectomy may have a potential survival benefit for patients with metastatic CRC. In our retrospective study, the status of mediastinal lymph nodes was a significant independent prognostic factor. Therefore, the presence or absence of mediastinal lymph node metastases must be accurately determined by thoracic lymph node dissection in cases where lymph node metastasis is suspected from preoperative evaluation and intraoperative findings. Also, a careful follow-up after the initial pulmonary metastasectomy is warranted, because at least one repeat pulmonary metastasectomy may improve the prognosis.

\section{Abbreviations \\ CRC: Colorectal cancer; JSCCR: Japanese Society for Cancer of the Colon and Rectum; CT: Computed tomography scan; PET: Positron emission tomography; CEA: Carcinoembryonic antigen; CA19-9: Carbohydrate antigen 19-9; DFI: Disease-free interval; VATS: Video-assisted thoracic surgery; \\ \%VC: Percent vital capacity; FEV $1.0 \%$ : Forced expiratory volume percent in $1 \mathrm{~s}$; Cls: Confidential intervals}

\section{Acknowledgments}

The authors thank the medical staff of the Department of Surgical Oncology and General and Cardiothoracic Surgery at Gifu University Hospital for their participation in this study. We could not have completed this study without their diligence and support. We would like to thank Editage (www.editage. jp) for the English language editing. 


\section{Authors' contributions}

MF conceived the study concept and planned the design as the principal investigator. MF interpreted the results and wrote the manuscript draft. NM, $\mathrm{HI}, \mathrm{KD}$, and $\mathrm{KY}$ revised the manuscript draft by adding intellectual insights and provided critical advice. MF, NM, TT, HY, KS, HI, KD, and KY obtained the data and provided their critical comments to improve the manuscript and gave final approval for submission. The author(s) read and approved the final manuscript.

\section{Funding}

None declared.

\section{Availability of data and materials}

The datasets used during this study are available from the corresponding author on reasonable request.

\section{Ethics approval and consent to participate}

The present study was conducted in accordance with the World Medical Association Declaration of Helsinki and was approved by the Ethics Committee of Gifu University (approval number '2019-253'). As this study was a retrospective study and did not include any potentially identifiable patient data, informed consent was not obtained from the enrolled patients. The institutional review board gave the ethics approval for this retrospective study.

\section{Consent for publication}

Not applicable.

\section{Competing interests}

K. Yoshida has received honoraria for lectures from Chugai Pharmaceutical Co., Ltd., Taiho Pharmaceutical Co., Ltd., Takeda Pharmaceutical Co., Ltd., Eli Lilly and Company, Daiichi Sankyo Co., Ltd., Ono Pharmaceutical Co., Ltd., Merck Serono Co., Ltd., Novartis Pharma K.K., and Sanofi K.K.; and research funding from Ajinomoto Pharmaceutical Co., Ltd., Takeda Pharmaceutical Co., Ltd., Chugai Pharmaceutical Co., Ltd., Daiichi Sankyo Co., Ltd., Taiho Pharmaceutical Co., Ono Pharmaceutical Co., and Yakult Honsha Co., Ltd. outside the submitted work.

T. Takahashi has received honoraria for lectures from Takeda Pharmaceutical Co., Ltd. All the remaining authors declare that they have no conflicts of interests.

\section{Author details}

'Department of Surgical Oncology, Graduate School of Medicine, Gifu University, 1-1 Yanagido, Gifu City, Gifu 501-1194, Japan. ${ }^{2}$ Department of General and Cardiothoracic Surgery, Graduate School of Medicine, Gifu University, 1-1 Yanagido, Gifu City, Gifu 501-1194, Japan.

Received: 16 August 2020 Accepted: 5 November 2020 Published online: 30 November 2020

\section{References}

1. McAfee MK, Allen MS, Trastek VF, Ilstrup DM, Deschamps C, Pairolero PC. Colorectal lung metastases: results of surgical excision. Ann Thorac Surg. 1992:53:780-5

2. Saito Y, Omiya H, Kohno K, Kobayashi T, Itoi K, Teramachi M, et al. Pulmonary metastasectomy for 165 patients with colorectal carcinoma: a prognostic assessment. J Thorac Cardiovasc Surg. 2002;124:1007-13.

3. Kanemitsu Y, Kato T, Hirai T, Yasui K. Preoperative probability model for predicting overall survival after resection of pulmonary metastases from colorectal cancer. Br J Surg. 2004;91:112-20.

4. Koga R, Yamamoto J, Saiura A, Yamaguchi T, Hata E, Sakamoto M. Surgical resection of pulmonary metastases from colorectal cancer: four favourable prognostic factors. Jpn J Clin Oncol. 2006;36:643-8.

5. lizasa T, Suzuki M, Yoshida S, Motohashi S, Yasufuku K, lyoda A, et al. Prediction of prognosis and surgical indications for pulmonary metastasectomy from colorectal cancer. Ann Thorac Surg. 2006;82: 254-60.

6. Watanabe K, Nagai K, Kobayashi A, Sugito M, Saito N. Factors influencing survival after complete resection of pulmonary metastases from colorectal cancer. Br J Surg. 2009;96:1058-65.
7. lida T, Nomori H, Shiba M, Nakajima J, Okumura S, Horio H, Metastatic Lung Tumor Study Group of Japan. Prognostic factors after pulmonary metastasectomy for colorectal cancer and rationale for determining surgical indications: a retrospective analysis. Ann Surg. 2013;257:1059-64.

8. Hirosawa T, Itabashi M, Ohnuki T, Yamaguchi N, Sugihara K, Kameoka S, Japanese Society for Cancer of the Colon and Rectum (JSCCR) Study Group for Pulmonary Metastases from Colorectal Cancer. Prognostic factors in patients undergoing complete resection of pulmonary metastases of colorectal cancer: a multi-institutional cumulative follow-up study. Surg Today. 2013:43:494-9.

9. Japanese Society for Cancer of the Colon and Rectum. Japanese Society for Cancer of the Colon and Rectum (JSCCR) Guidelines 2019 for the Treatment of Colorectal cancer. Int J Clin Oncol. 2020:25:1-42.

10. Brierley JD, Gospodarowicz MK, Wittekind C. International Union Against Cancer. TNM Classification of Malignant Tumors. 8th ed. Wiley-Blackwell: Chichester; 2017.

11. Clavien PA, Barkun J, de Oliveira ML, Vauthey JN, Dindo D, Schulick RD, et al The Clavien-Dindo classification of surgical complications: five-year experience. Ann Surg. 2009;250:187-96.

12. Parkin DM, Bray F, Ferlay J, Pisani P. Global Cancer Statistics, 2002. CA Cancer J Clin. 2005:55:74-108.

13. Bray F, Ferlay J, Soerjomataram I, Siegel RL, Torre LA, Jemal A. Global cancer statistics 2018: GLOBOCAN estimates of incidence and mortality worldwide for 36 cancers in 185 countries. CA Cancer J Clin. 2018;68: 394-424.

14. Thomford NR, Woolner LB, Clagett OT. The surgical treatment of metastatic tumors in the lungs. J Thrac Cardiovasc Surg. 1965;49:357-63.

15. Treasure T, Farewell V, Macbeth F, Monson F, Williams NR, Brew-Graves C, et al. Pulmonary metastasectomy versus continued active monitoring in colorectal cancer (PulMiCC): a multicentre randomized clinical trial. Trials. 2019;20:718.

16. Brown KGM, Koh CE. Surgical management of recurrent colon cancer. J Gastrointest Oncol. 2020;11:513-25.

17. Okumura $\mathrm{S}$, Kondo $\mathrm{H}$, Tsuboi $\mathrm{M}$, Nakayama $\mathrm{H}$, Asamura $\mathrm{H}$, Tsuchiya $\mathrm{R}$, et al. Pulmonary resection for metastatic colorectal cancer: experiences with 159 patients. J Thorac Cardiovasc Surg. 1996;112:867-74.

18. Kandioler D, Krömer E, Tüchler H, End A, Müller MR, Wolner E, et al. Longterm results after repeated surgical removal of pulmonary metastases. Ann Thorac Surg. 1998;65:909-12.

19. Inoue $M$, Ohta $M$, luchi $K$, Matsumura $A$, Ideguchi $K$, Yasumitsu $T$, et al. Benefits of surgery for patients with pulmonary metastases from colorectal carcinoma. Ann Thorac Surg. 2004;78:238-44.

20. Pfannschmidt J, Dienemann $H$, Hoffmann $H$. Surgical resection of pulmonary metastases from colorectal cancer: a systematic review of published series. Ann Thorac Surg. 2007;84:324-38.

21. Kanzaki R, Higashiyama M, Oda K, Fujiwara A, Tokunaga T, Maeda J, et al. Outcome of surgical resection for recurrent pulmonary metastasis from colorectal carcinoma. Am J Surg. 2011;202:419-26.

22. Gonzalez M, Poncet A, Combescure C, Robert J, Ris HB, Gervaz P. Risk factors for survival after lung metastasectomy in colorectal cancer patients: a systematic review and meta-analysis. Ann Surg Oncol. 2013; 20:572-9.

23. Jaklitsch MT, Mery CM, Lukanich JM, Richards WG, Bueno R, Swanson SJ, et al. Sequential thoracic metastasectomy prolongs survival by reestablishing local control within the chest. J Thorac Cardiovasc Surg. 2001; 121:657-67.

24. Fiorentino F, Hunt I, Teoh K, Treasure T, Utley M. Pulmonary metastasectomy in colorectal cancer: a systematic review and quantitative synthesis. J R Soc Med. 2010;103:60-6.

25. Ampollini L, Gnetti L, Goldoni M, Viani L, Faedda E, Campanini N, et al. Pulmonary metastasectomy for colorectal cancer: analysis of prognostic factors affecting survival. J Thorac Dis. 2017;12:1282-90.

26. Cho JH, Kim S, Namgung M, Choi YS, Kim HK, Zo Jl, et al. The prognostic importance of the number of metastases in pulmonary metastasectomy of colorectal cancer. World J Surg Oncol. 2015;13:222.

27. Nanji S, Karim S, Tang E, Brennan K, McGuire A, Pramesh CS, et al. Pulmonary metastasectomy for colorectal cancer: predictors of survival in routine surgical practice. Ann Thorac Surg. 2018;105:1605-12.

28. Hamaji M, Cassivi SD, Shen KR, Allen MS, Nichols FC, Deschamps C, et al. Is lymph node dissection required in pulmonary metastasectomy for colorectal adenocarcinoma? Ann Thorac Surg. 2012;94:1796-800. 
29. Welter S, Jacobs J, Krbek T, Poettgen C, Stamatis G. Prognostic impact of lymph node involvement in pulmonary metastases from colorectal cancer. Euro J Cardiothoracic Surg. 2007;31:167-72.

30. Hachimaru A, Maeda R, Suda T, Takagi Y. Repeat pulmonary resection for recurrent lung metastases from colorectal cancer: an analysis of prognostic factors. Interact Cardiovasc Thorac Surg. 2016;22:826-30.

31. Ogata Y, Matono K, Hayashi A, Takamor S, Miwa K, Sasatomi T, et al. Repeat pulmonary resection for isolated recurrent lung metastases yields results comparable to those after first pulmonary resection in colorectal cancer. World J Surg. 2005;29:363-8.

32. Chen F, Sakai H, Miyahara R, Bando T, Okubo K, Date H. Repeat resection of pulmonary metastasis is beneficial for patients with colorectal carcinoma. World J Surg. 2010;34:2373-8.

33. Murakawa T. Past, present, and future perspectives of pulmonary metastasectomy for patients with advanced colorectal cancer. Surg Today. 2020. https://doi.org/10.1007/s00595-020-02119-y.

\section{Publisher's Note}

Springer Nature remains neutral with regard to jurisdictional claims in published maps and institutional affiliations.

Ready to submit your research? Choose BMC and benefit from:

- fast, convenient online submission

- thorough peer review by experienced researchers in your field

- rapid publication on acceptance

- support for research data, including large and complex data types

- gold Open Access which fosters wider collaboration and increased citations

- maximum visibility for your research: over $100 \mathrm{M}$ website views per year

At BMC, research is always in progress.

Learn more biomedcentral.com/submissions 